\title{
GROTHENDIECK GROUPS OF TWISTED FREE ASSOCIATIVE ALGEBRAS
}

\author{
by KOO-GUAN CHOO
}

(Received 1 April, 1976)

Let $R$ be an associative ring with identity, $X$ a set of noncommuting variables, $\mathscr{A}=\left\{\alpha_{x}\right\}_{x \in X}$ a set of automorphisms $\alpha_{x}$ of $R$ and $R_{\mathscr{A}}\{X\}$ the $\mathscr{A}$-twisted free associative algebra on $X$ over $R$. Let $Y$ be another set of noncommuting variables, $\mathscr{B}=\left\{\beta_{y}\right\}_{y \in Y}$ a set of automorphisms $\beta_{y}$ of $R_{\mathscr{A}}\{X\}$ and $S=\left(R_{\mathscr{S}}\{X\}\right)_{\mathscr{B}}\{Y\}$ the $\mathscr{B}$-twisted free associative algebra on $Y$ over $R_{\mathscr{A}}\{X\}$. Next, let $X_{l}$ be a set of noncommuting variables, for each $l=1,2, \ldots$ We form the free associative algebra $S_{1}=S\left\{X_{1}\right\}$ on $X_{1}$ over $S$ and inductively, we form the free associative algebra $S_{l+1}=S_{l}\left\{X_{l+1}\right\}$ on $X_{l+1}$ over $S_{l}, l=1,2, \ldots$. The main purpose of the paper is to prove that if $R$ is a right Noetherian ring of finite right global dimension, then (a) $K_{0} R$ and $K_{0} R_{\mathscr{s}}\{X\}$ are isomorphic; (b) $K_{n} R$ and $K_{n} S$ are isomorphic, $n=0,1$; and (c) $K_{n} R$ and $K_{n} S_{l}$ $(n=0,1)$ are isomorphic, for each $l=1,2, \ldots$

1. Statements of main theorems. Let $R$ be an associative ring with identity. We denote the Grothendieck group of $R$ by $K_{0} R$ and the Whitehead group of $R$ by $K_{1} R$.

We recall the definition of twisted free associative algebras. For undefined terminologies, we refer to [4] and [2].

Let $R$ be an associative ring with identity. Let $X$ be a set of noncommuting variables and $\mathscr{A}=\left\{\alpha_{x}\right\}_{x \in X}$ a set of automorphisms $\alpha_{x}$ of $R$. The $\mathscr{A}$-twisted free associative algebra on $X$ over $R$, denoted by $R_{\mathscr{A}}\{X\}$, is defined as follows: additively, $R_{\mathscr{A}}\{X\}=R\{X\}$ so that its elements are finite linear combinations of words $w(x)$ in $x \in X$ with coefficients in $R$; if $w(x)=x_{1} \ldots x_{n}$ is a word in $x_{1}, \ldots, x_{n}$, we denote the automorphism $\alpha_{x_{1}} \ldots \alpha_{x_{n}}$ by $w(\alpha) ;$ multiplication in $R_{\mathscr{A}}\{X\}$ is given by

$$
(r w(x))\left(r^{\prime} w^{\prime}(x)\right)=r w(\alpha)^{-1}\left(r^{\prime}\right) w(x) w^{\prime}(x),
$$

for any $r w(x), r^{\prime} w^{\prime}(x)$ in $R_{\mathscr{d}}\{X\}$.

In particular, if $X=\{t\}$ and $\mathscr{A}=\{\alpha\}$, then $R_{\mathscr{A}}\{X\}$ is just the $\alpha$-twisted polynomial ring $R_{\alpha}[t]$.

We shall consider $R_{\mathscr{A}}\{X\}$ as an $R$-ring with augmentation $\varepsilon_{X}: R_{\mathscr{A}}\{X\} \rightarrow R$ defined by $\varepsilon_{X}(x)=0$ for each $x \in X$. Then the inclusion map $i: R \rightarrow R_{\mathscr{A}}\{X\}$ induces a one-to-one homomorphism $i_{*}: K_{n} R \rightarrow K_{n} R_{\mathscr{A}}\{X\}, n=0,1$.

In [1, Theorem 2], we have shown that if $K_{1} R \rightarrow K_{1} R_{\alpha}[t]$ is an isomorphism for certain automorphisms $\alpha$ of $R$, then $K_{1} R \rightarrow K_{1} R_{\mathscr{A}}\{X\}$ is an isomorphism. Farrell has shown in [3, Theorem 1.6] that if $R$ is a right Noetherian ring of finite right global dimension, then $K_{1} R \rightarrow$ $K_{1} R_{\alpha}[t]$ is an isomorphism, for any automorphism $\alpha$ of $R$. Hence if $R$ is a right Noetherian ring of finite right global dimension, then $K_{1} R \rightarrow K_{1} R_{\mathscr{A}}\{X\}$ is an isomorphism. The first purpose of this paper is to show the analogous result for $K_{0}$.

Glasgow Math. J. 18 (1977) 193-196. 
THEOREM 1. Let $R$ be a right Noetherian ring of finite right global dimension. Then the inclusion map $i: R \rightarrow R_{\mathscr{A}}\{X\}$ induces an isomorphism $i_{*}: K_{0} R \rightarrow K_{0} R_{\mathscr{S}}\{X\}$.

We remark that for a (non-twisted) free associative algebra, this is contained in [5, Corollary 3.9].

Now let $Y$ be another set of noncommuting variables, $\mathscr{B}=\left\{\beta_{y}\right\}_{y \in Y}$ a set of automorphisms $\beta_{y}$ of $R_{\mathscr{A}}\{X\}$, and $S=\left(R_{\mathscr{A}}\{X\}\right)_{\mathscr{D}}\{Y\}$ the $\mathscr{B}$-twisted free associative algebra on $Y$ over $R_{\mathscr{A}}\{X\}$. We have the natural inclusion maps $j: R_{\mathscr{A}}\{X\} \rightarrow S$ and $k: R \rightarrow S$. Then we show:

Theorem 2. Let $R$ be a right Noetherian ring of finite right global dimension. Then the inclusion map $k: R \rightarrow S$ induces an isomorphism $k_{*}: K_{n} R \rightarrow K_{n} S, n=0,1$.

Next, let $X_{l}$ be a set of noncommuting variables, for each $l=1,2, \ldots$ We form the free associative algebra $S_{1}=S\left\{X_{1}\right\}$ on $X_{1}$ over $S$, where $S$ is defined as above, and inductively, we form the free associative algebra $S_{l+1}=S_{l}\left\{X_{l+1}\right\}$ on $X_{l+1}$ over $S_{l}, l=1,2, \ldots$ That is, for each $l=1,2, \ldots, S_{l}$ is the ring of the form

$$
\left.S_{l}=\left(\ldots\left(\left(R_{\mathscr{A}}\{X\}\right)_{\mathscr{g}}\{Y\}\right)\left\{X_{1}\right\}\right) \ldots\right)\left\{X_{l}\right\} .
$$

We note that the polynomial ring $S_{l}[t]$ is canonically isomorphic to

$$
S[t]_{l}=\left(\ldots\left(\left(\left(R[t]_{\mathscr{A}}\{X\}\right)_{\mathscr{Q}}\{Y\}\right)\left\{X_{1}\right\}\right) \ldots\right)\left\{X_{l}\right\},
$$

$(l=1,2, \ldots)$. Then we extend the results of Theorem 2 to:

THEOREM 3. Let $R$ be a right Noetherian ring of finite right global dimension. Then $K_{n} R$ and $K_{n} S_{l}(n=0,1)$ are isomorphic for $l=1,2, \ldots$

2. Some known results. In this section, we collect some results which will be used in the proof of the theorems. First, we recall the following result of Farrell and Hsiang [4, Lemmas 23 and 24].

LEMMA 4. If $R$ is a right Noetherian ring of finite right global dimension, then the twisted polynomial ring $R_{\alpha}[t]$ and the twisted group ring $R_{\alpha}[T]$ are right Noetherian and of finite right global dimension, where $T$ denotes an infinite cyclic group.

We have observed in [2] that a modification of the proof of Farrell's result [3, Theorem 1.6] gives:

LEMMA 5. If $R$ is a right coherent ring of finite right global dimension, then the inclusion map $R \rightarrow R_{\alpha}[t]$ induces an isomorphism $K_{1} R \rightarrow K_{1} R_{\alpha}[t]$.

Hence it is immediate from this lemma and [1. Theorem 2] that:

Proposition 6. Let $R$ be a right coherent ring of finite right global dimension. Then the inclusion map $R \rightarrow R_{\mathscr{A}}\{X\}$ induces an isomorphism $K_{1} R \rightarrow K_{1} R_{\mathscr{A}}\{X\}$.

Also, it is clear from [4, Theorems 13 and 19] that:

Proposition 7. Let $R$ be a ring such that $K_{1} R \cong K_{1} R[t]$ (in particular, let $R$ be a right coherent ring of finite right global dimension). Let $T$ be an infinite cyclic group and $R[T]$ the group ring of $T$ over $R$. Then $K_{1} R[T] \cong K_{1} R \oplus K_{0} R$. 
It was proved in [2] and [5] that if $R$ is a right Noetherian ring of finite right global dimension, then the free associative algebra $R\{X\}$ is right coherent and of finite right global dimension. In fact, using Lemma 4 and [2, Theorem 2.1] (cf. [5, Proposition 1.9]), we have:

Proposition 8. Let $R$ be a right Noetherian ring of finite right global dimension. Then the $\mathscr{A}$-twisted free associative algebra $R_{\mathscr{A}}\{X\}$ is right coherent and of finite right global dimension.

3. Proofs of main theorems. Now we give the proof of our theorems.

Proof of Theorem 1. Since $R$ is right Noetherian and of finite right global dimension, $R_{s}\{X\}$ is right coherent and of finite right global dimension by Proposition 8. Thus, by Proposition $7, K_{1}\left(R_{\mathscr{S}}\{X\}\right)[T] \cong K_{1} R_{\mathscr{S}}\{X\} \oplus K_{0} R_{\mathscr{A}}\{X\}$. Now, we note that $\left(R_{\mathscr{S}}\{X\}\right)[T]$ is canonically isomorphic to $(R[T])_{\mathscr{A}}\{X\}$ and since $R[T]$ is right Noetherian and of finite right global dimension, by Lemma 4, it follows from Proposition 6 that $K_{1} R[T] \cong K_{1}(R[T])_{\mathcal{A}}\{X\}=$ $K_{1}\left(R_{\mathscr{A}}\{X\}\right)[T]$. Thus $K_{1} R[T] \cong K_{1} R_{\mathscr{A}}\{X\} \oplus K_{0} R_{\mathscr{S}}\{X\}$. But $K_{1} R[T] \cong K_{1} R \oplus K_{0} R$ by Proposition 7 and $K_{1} R_{s}\{X\} \cong K_{1} R$ by Proposition 6. Hence $K_{1} R \oplus K_{0} R \cong K_{1} R \oplus K_{0} R_{\mathscr{A}}\{X\}$. Since the composite isomorphism carries $K_{1}$ terms to $K_{1}$ terms and $K_{0}$ terms to $K_{0}$ terms, we deduce that $K_{0} R \cong K_{0} R_{\mathscr{S}}\{X\}$. This completes the proof.

Proof of Theorem 2. Since $R$ is right Noetherian and of finite right global dimension, the inclusion map $i: R \rightarrow R_{\mathscr{A}}\{X\}$ induces an isomorphism $i_{*}: K_{1} R \rightarrow K_{1} R_{\mathscr{A}}\{X\}$ by Proposition 6 . Now $R_{s}\{X\}$ is right coherent and of finite right global dimension by Proposition 8 , so that the inclusion map $j: R_{s}\{X\} \rightarrow S$ induces an isomorphism $j_{*}: K_{1} R_{s}\{X\} \rightarrow K_{1} S$, again by Proposition 6. Hence the inclusion map $k: R \rightarrow S$ induces an isomorphism $k_{*}: K_{1} R \rightarrow K_{1} S$.

Next, we note that $S[t]$ is canonically isomorphic to $\left(R[t]_{\mathscr{A}}\{X\}\right)_{\mathscr{B}}\{Y\}$. Since $R[t]$ is right Noetherian and of finite right global dimension by Lemma 4 , it follows from the first part of the proof that $K_{1} S[t] \cong K_{1}\left(R[t]_{\mathscr{A}}\{X\}\right)_{\mathscr{Q}}\{Y\} \cong K_{1} R[t]$. But $K_{1} R[t] \cong K_{1} R$ by Lemma 5 and $K_{1} S \cong K_{1} R$, thus $K_{1} S \cong K_{1} S[t]$. Hence, by Proposition $7, K_{1} S[T] \cong K_{1} S \oplus K_{0} S$, where $S[T]$ is the group ring of an infinite cyclic group $T$ over $S$. Finally, as in the proof of Theorem 1, we have

$$
\begin{aligned}
K_{1} S[T] & \cong K_{1}\left(R[T]_{\mathscr{\infty}}\{X\}\right)_{\mathscr{\infty}}\{Y\} \\
& \cong K_{1} R[T] \quad \text { (first part of the proof) } \\
& \cong K_{1} R \oplus K_{0} R \quad \text { (Proposition 7) }
\end{aligned}
$$

Hence $K_{1} S \oplus K_{0} S \cong K_{1} R \oplus K_{0} R$. Since $K_{1} S \cong K_{1} R$, and since the composite isomorphism carries $K_{1}$ terms to $K_{1}$ terms and $K_{0}$ terms to $K_{0}$ terms, therefore $K_{0} S \cong K_{0} R$. This completes the proof.

Proof of Theorem 3. We prove the result by induction on $l$ for $K_{1}$.

As contained in the proof of Theorem 2, we have shown that $K_{1} S$ and $K_{1} S[t]$ are isomorphic, where $S[t]$ is the polynomial ring in $t$ over $S$. Thus, it follows immediately from this fact and the Gersten theorem on (non-twisted) free associative algebra (cf. [1, Theorem 2]) that $K_{1} S$ and $K_{1} S_{1}$ are isomorphic. Hence, by Theorem 2, $K_{1} R$ and $K_{1} S_{1}$ are isomorphic. This starts the induction. 
Now suppose that, for a right Noetherian ring $R$ of finite right global dimension, $K_{1} R \cong K_{1} S_{m}$ for some $l=m$. Since $R[t]$ is right Noetherian and of finite right global dimension, by the inductive hypothesis,

$$
K_{1} R[t] \cong K_{1} S[t]_{m}
$$

where $S[t]_{m}$ is given by (1). Since $S_{m}[t] \cong S[t]_{m}$ and $K_{1} R \cong K_{1} R[t]$, therefore $K_{1} R \cong K_{1} S_{m}[t]$ so that $K_{1} S_{m} \cong K_{1} S_{m}[t]$. Again, by using the Gersten theorem on free associative algebra, we conclude that $K_{1} S_{m} \cong K_{1} S_{m}\left\{X_{m+1}\right\}=K_{1} S_{m+1}$. Hence $K_{1} R \cong K_{1} S_{m+1}$. This finishes the proof that $K_{1} R \cong K_{1} S_{l}$ for $l=1,2, \ldots$.

A similar argument as in the proof of Theorem 1 gives $K_{0} R \cong K_{0} S_{l}$ for $l=1,2, \ldots$ and this completes the proof.

\section{REFERENCES}

1. Koo-Guan Choo, Whitehead groups of twisted free associative algebras, Pacific J. Math. 50 (1974), 399-402.

2. K. G. Choo, K. Y. Lam and E. Luft, On free product of rings and the coherence property, Lectures Notes in Mathematics 342 (Springer-Verlag, 1973), 135-143.

3. F. T. Farrell, The obstruction of fibering a manifold over a circle, Indiana Univ. Math. J. 21 (1971), 315-346.

4. F. T Farrell and W. C. Hsiang, A formula for $K_{1} R_{a}[T]$, Proc. Sympos. Pure Math. 17 (Amer. Math. Soc., 1970), 192-218.

5. S. M. Gersten, K-theory of free rings, Comm. in Algebra 1(1) (1974), 39-64.

Department of Pure Mathematics

UNIVERSITY OF SYDNEY

SYDNEY, N.S.W. 2006

Australia 